\title{
Diffusive-Ballistic Transition in Random Walks with Long-Range Self-Repulsion
}

\author{
Aldo Procacci, Rémy Sanchis, Benedetto Scoppola
}

November 21, 2018

\begin{abstract}
We prove that a class of random walks on $\mathbb{Z}^{2}$ with long-range selfrepulsive interactions have a diffusive-ballistic phase transition.
\end{abstract}

MSC Numbers: 82B20 82B41 82B26

Keywords: Self-Repelling Random Walks, One-dimesional Ising model, Long Range Interactions, Diffusive-Ballistic Phase Transition.

\section{$\S 1$. Introduction, model and results}

Random walks are a simple mathematical tool to describe polymers, which are the subject of study of a rapidly developing area intersecting physics, chemistry and recently biophysics. In particular, random walks with self-repulsive long range interactions are a natural model for polyelectrolytes (see e.g. [7]).

In this note we propose a model of random walks with long-range self-repulsion exhibiting a diffusive-ballistic phase transition. Our arguments stem from the ideas illustrated in a previous paper [4] where it was shown that a polymer in two dimensions with a self repelling interaction of Kac type exhibits a diffusiveballistic transition if considered on the appropriate scale. Here we prove that the reasoning and conclusion of [1] can be generalized to infinite range Coulomb-like interactions (i.e. decaying polynomially) and in this case the phase transition is a genuine one, namely it does not depend on some length scale as in [4].

Self-repulsive random walks with long range interactions are not new in the literature. E.g. random walk models with some similarity to the model proposed here has been studied in [3], [12] and [5]. See also [10] for rigorous results on a similar model.

The class of random walks in $\mathbb{Z}^{2}$ that we consider in this paper, denoted $\mathcal{W}_{N}$, is described as a succession of $N$ steps, $\omega=\left(\omega_{0}, \omega_{1}, \ldots \omega_{N}\right)$, with $\omega_{i} \in \mathbb{Z}^{2}$ and 
$\left|\omega_{i+1}-\omega_{i}\right|=1$, starting at the origin, $\omega_{0}=0$ and weighted according to

$$
P_{\beta}(\omega)=Z_{N}^{-1} \exp \left[+\beta \sum_{0 \leq i<j \leq N} V_{i j} \cdot\left(\omega_{i}-\omega_{j}\right)^{2}\right]
$$

where $\beta>0$ is a parameter which plays the role of the inverse temperature and $Z_{N}$ is the normalizing factor given by

$$
Z_{N}(\beta)=\sum_{\omega \in \mathcal{W}_{N}} \exp \left(+\beta \sum_{0 \leq i<j \leq N} V_{i j} \cdot\left(\omega_{i}-\omega_{j}\right)^{2}\right)
$$

The self interaction $V_{i j}$, decreasing with the difference of the proper time $i$ and $j$ of the random walk, has the following form:

$$
V_{i j}=\frac{1}{|i-j|^{\alpha}} \quad 3<\alpha \leq 4
$$

These weights assign greater probability to walks that are "far apart" or are stiffer.

Let

$$
\left\langle\omega_{N}^{2}\right\rangle=\sum_{\omega \in \mathcal{W}_{\mathcal{N}}} \omega_{N}^{2} P_{\beta}(\omega)
$$

be the mean square end-to-end distance of the walk $\omega$ and let $\gamma>0$ such that $\lim _{N \rightarrow \infty}\left\langle\omega_{N}^{2}\right\rangle / N^{\gamma}$ is finite positive. A random walk is said to be diffusive if $\gamma=1$, superdiffusive if $1<\gamma<2$ and ballistic if $\gamma=2$.

We will show that the self repelling random walk model proposed in this note is diffusive at sufficiently high temperature and ballistic at suffciently low temperatures. Our result can be summarized by the following theorem.

Theorem. Consider the random walk defined in (1)-(3). There exist positive numbers $\beta_{1}, \beta_{2}\left(\beta_{1}>\beta_{2}\right), C_{1}$ and $C_{2}$ such that

$$
\left\langle\omega_{N}^{2}\right\rangle \geq C_{1} N^{2}, \quad \text { for all } \beta>\beta_{1}
$$

and

$$
\left\langle\omega_{N}^{2}\right\rangle \leq C_{2} N, \quad \text { for all } \beta<\beta_{2}
$$

Remark. Theorem above implies immediately that our random walk model has two distinct regimes for the behavior of the end-to-end distance. Namely, inequality (5) implies that the model defined by (1)-(3) is ballistic (i.e. $\gamma=2$ ) for all inverse temperature above $\beta_{1}$ and inequality (6) implies that the same model is diffusive (i.e. $\gamma=1$ ) for all inverse temperature below $\beta_{2}$. 
Observe that the theorem says nothing about the behavior of the end-to-end distance with $N$ at inverse temperature in the interval $\beta \in\left[\beta_{2}, \beta_{1}\right]$. So, in principle, the existence of an intermediate super-diffusive phase for the present model in the region $\beta \in\left[\beta_{2}, \beta_{1}\right]$ cannot be excluded.

\section{§2. Proof of the theorem}

To prove the theorem above, following the ideas of [4], we first define two further changes of variables. Let first $\mu_{i}=\omega_{i}-\omega_{i-1}$, for $i=1, \ldots, N$. Then $\mu_{i} \in$ $\left\{ \pm e_{1}, \pm e_{2}\right\}$, where $e_{1}=(1,0)$ and $e_{2}=(0,1)$. Moreover $\omega_{k}=\sum_{i=1}^{k} \mu_{i}$. We now decompose the vectors $\mu_{i}$ in the following way

$$
\mu_{i}=\sigma_{i} \frac{e_{1}+e_{2}}{\sqrt{2}}+\tilde{\sigma}_{i} \frac{e_{1}-e_{2}}{\sqrt{2}}
$$

with $\sigma_{i}, \tilde{\sigma}_{i} \in\{ \pm 1\}$. We will denote a set of $\sigma_{i}, \tilde{\sigma}_{i}, \quad, 1 \leq i \leq N$ as $\sigma, \tilde{\sigma}$. It is easy to see that the correspondence between a succession $\omega \in \mathcal{W}_{\mathcal{N}}$ and a set $\sigma, \tilde{\sigma}$ is one-to-one and the probability measure $P(\omega)$ is mapped in

$$
P_{\beta}(\omega)=P_{\beta}(\mu)=P_{\beta}(\sigma, \tilde{\sigma})=P_{\beta}(\sigma) P_{\beta}(\tilde{\sigma})
$$

with

$$
P_{\beta}(\sigma)=\frac{\exp \left(+\beta \sum_{0 \leq i<j \leq N} U_{i j}^{N} \sigma_{i} \sigma_{j}\right)}{\sum_{\sigma \in \Sigma_{N}} \exp \left(+\beta \sum_{0 \leq i<j \leq N} U_{i j}^{N} \sigma_{i} \sigma_{j}\right)}
$$

and

$$
U_{i j}^{N}=\sum_{0 \leq k \leq i<j \leq l \leq N} V_{k l}
$$

Moreover observe that

$$
\omega_{N}^{2}=\left(\sum_{i=1}^{N} \mu_{i}\right)^{2}=\sum_{i=1}^{n} \sum_{j=1}^{n} \mu_{i} \mu_{j}=\sum_{i=1}^{n} \sum_{j=1}^{n}\left(\sigma_{i} \sigma_{j}+\tilde{\sigma}_{i} \tilde{\sigma}_{j}\right)
$$

Hence we can rewrite (4) as

$$
\begin{gathered}
\left\langle\omega_{N}^{2}\right\rangle=\sum_{\omega \in \mathcal{W}_{\mathcal{N}}} \omega_{N}^{2} P_{\beta}(\omega)=\sum_{\sigma \in \Sigma_{N}} \sum_{\tilde{\sigma} \in \Sigma_{N}} \sum_{0 \leq i, j \leq N}\left(\sigma_{i} \sigma_{j}+\tilde{\sigma}_{i} \tilde{\sigma}_{j}\right) P_{\beta}(\sigma) P_{-} \beta(\tilde{\sigma})= \\
=2 \sum_{0 \leq i, j \leq N} \sum_{\sigma \in \Sigma_{N}} \sigma_{i} \sigma_{j} P_{\beta}(\sigma)=2 \sum_{0 \leq i, j \leq N}\left\langle\sigma_{i} \sigma_{j}\right\rangle_{\mathcal{N}}^{\beta U^{N}}
\end{gathered}
$$


Therefore we have shown that the mean value of the square end-to-end distance of the self-repelling random walk in $\mathbb{Z}^{2}$ is twice the mean value of $\sum_{0 \leq i, j \leq N} \sigma_{i} \sigma_{j}$ in a one dimensional spin system on $\mathcal{N}=\{1,2, \ldots, N\}$ at inverse-temperature $\beta$ with free boundary conditions and ferromagnetic Hamiltonian given by

$$
H_{\mathcal{N}}^{0}\left(\sigma_{\mathcal{N}}\right)=-\sum_{1 \leq i<j \leq N} U_{i j}^{N} \sigma_{i} \sigma_{j}
$$

Note that the spin potential $U_{i j}^{N}$ defined in (8) has some dependence on the volume $N$. However, since $V_{i j}=|i-j|^{-\alpha}$, it is easy to show that for a fixed $\varepsilon>0$, and all $i, j \in[\varepsilon N,(1-\varepsilon) N]$, there are constants $c_{1}, c_{2}$ depending on $\varepsilon$ such that

$$
\bar{U}_{i j}^{(1)}=c_{1}|i-j|^{-\alpha+2} \leq U_{i j}^{N} \leq c_{2}|i-j|^{-\alpha+2}=\bar{U}_{i j}^{(2)}
$$

On the other hand, when $i, j$ are not both inside the region $\mathcal{N}_{\varepsilon}=\{i \in \mathcal{N}: \varepsilon N \leq$ $i \leq(1-\varepsilon) N\}$, the potential $U_{i j}^{N}$, that is evidently ferromagnetic everywhere, can be bounded by

$$
0 \leq U_{i j}^{N} \leq c_{2}|i-j|^{-\alpha+2}
$$

Using now the Griffiths inequalities (valid for ferromegnetic systems) we have the following bounds for the correlations.

$$
\left\langle\sigma_{i} \sigma_{j}\right\rangle_{\mathcal{N}_{\varepsilon}}^{\beta \bar{U}^{(1)}} \leq\left\langle\sigma_{i} \sigma_{j}\right\rangle_{\mathcal{N}^{N}}^{\beta U^{N}} \leq\left\langle\sigma_{i} \sigma_{j}\right\rangle_{\mathcal{N}}^{\beta \bar{U}^{(2)}}
$$

The inequality (13) is crucial for the purposes of this note since it easily implies the diffusive-ballistic transition of our model. It states that the quantity $\left\langle\sigma_{i} \sigma_{j}\right\rangle_{\mathcal{N}}^{\beta U^{N}}$, directly related to the mean end-to-end distance via (9), is bounded above the by the free boundary condition spin-spin correlation function of a onedimensional spin system in the volume $\mathcal{N}$ with Hamiltonian given by

$$
H_{\mathcal{N}}^{2}=-\sum_{\substack{i<j \\\{i, j\} \in \mathcal{N}}} \frac{c_{2}}{|i-j|^{\alpha-2}} \sigma_{i} \sigma_{j}
$$

The inequality (13) also states that the same quantity $\left\langle\sigma_{i} \sigma_{j}\right\rangle_{\mathcal{N}}^{\beta U^{N}}$ is bounded below by the free boundary condition spin-spin (not truncated) correlation of an analogous one-dimensional spin system, this time in the smaller volume $\mathcal{N}_{\varepsilon}$ with Hamiltonian given by

$$
H_{\mathcal{N}}^{1}=-\sum_{\substack{i<j \\\{i, j\} \in \mathcal{N}}} \frac{c_{1}}{|i-j|^{\alpha-2}} \sigma_{i} \sigma_{j}
$$

Now, it is a consequence of [6] and [8] that, if $3<\alpha \leq 4$, there exists a value $\beta_{1}$ (depending on $c_{1}$ ) such that the spin system with Hamiltonian (15) has 
spontaneous magnetization $m_{*}(\beta)>0$ for all $\beta>\beta_{1}$. By [11], the infinite volume limit of the two point function $\left\langle\sigma_{i} \sigma_{j}\right\rangle_{\mathcal{N}_{\varepsilon}}^{\beta \bar{U}^{(1)}}$ with free boundary condition converges to $m_{*}^{2}$. In other words, for $N$ sufficiently large,

$$
\left\langle\sigma_{i} \sigma_{j}\right\rangle_{\mathcal{N}_{\varepsilon}}^{\beta \bar{U}^{(1)}} \geq \frac{1}{2} m_{*}^{2}(\beta), \quad \text { for all } \beta>\beta_{1}
$$

Therefore, using the lower bounds (13) and (16)and recalling that $m(\beta)$ is monotonic increasing with $\beta$, we get, for $N$ sufficiently large, and for any $\beta>\beta_{1}$

$$
\left\langle\omega_{N}^{2}\right\rangle=2 \sum_{0 \leq i, j \leq N}\left\langle\sigma_{i} \sigma_{j}\right\rangle_{\mathcal{N}}^{\beta U^{N}} \geq C_{1} N^{2}
$$

where $C_{1}=(1-2 \varepsilon)^{2} m_{*}^{2}\left(\beta_{1}\right)$. This proves inequality (5).

Concerning now the upper bound for $\left\langle\sigma_{i} \sigma_{j}\right\rangle_{\mathcal{N}}^{\beta \bar{U}^{(2)}}$, again we can use the classical results [6] and [8] to claim that, if $3<\alpha \leq 4$, there exists an inverse temperature $\beta_{2}$ (and $\beta_{2}<\beta_{1}$, since $c_{2}>c_{1}$ ) such that the spin system with Hamiltonian (14), for all $\beta<\beta_{2}$, has no spontaneous magnetization (i.e. $m_{*}=0$ ) and, by [1] (see also [2] and [14]), there exists a positive constant $0<C_{\beta_{2}}<+\infty$ such that the infinite volume limit of the two point function $\left\langle\sigma_{i} \sigma_{j}\right\rangle_{\mathcal{N}_{\varepsilon}}^{\beta \bar{U}^{(2)}}$ with free boundary condition is bounded above by $C_{\beta_{2}}|i-j|^{-\alpha+2}$. In other words, for $N$ sufficiently large

$$
\left\langle\sigma_{i} \sigma_{j}\right\rangle_{\mathcal{N}_{\varepsilon}}^{\beta \bar{U}^{(2)}} \leq C_{\beta_{2}} \frac{1}{|i-j|^{\alpha-2}}, \quad \text { for all } \beta<\beta_{2}
$$

Thus, using the upper bounds (13) and (18), we get, for $N$ sufficiently large, we get that

$$
\left\langle\omega_{N}^{2}\right\rangle=2 \sum_{0 \leq i, j \leq N}\left\langle\sigma_{i} \sigma_{j}\right\rangle_{\mathcal{N}}^{\beta U^{N}} \leq C_{2} N
$$

whenever $\beta<\beta_{2}$, with $C_{2}=2 C_{\beta_{2}} \sum_{i>0} i^{-\alpha+2}$. This proves inequality (6) and concludes the proof of the theorem.

\section{§3. Concluding Remarks.}

As remarked above, the comparison method proposed in this note, based on the inequalities (11)-(13), doesn't allow to conclude that the random walk model defined by (1)-(3) exhibits a genuine diffusive-ballistic phase transition with a unique critical point $\beta_{s}$ inside the interval $\left[\beta_{1}, \beta_{2}\right]$ below which the system is diffusive and above which the system is ballistic. In order to prove that, one should study directly the Ising model with Hamiltonian (10) and show that results about the standard Ising model with Hamiltonian (14) remain valid. In particular, one should prove that the two point correlation function is absolutely summable in 
the whole subcritical phase. This task does not seem completely trivial since Hamiltonian (10), differently from (14), is not translational invariant.

The theorem above could be generalized for random walks described by (1)(3) with any power $\alpha \in \mathbb{R}^{+}$. As a matter of fact, by the results contained in [13], it is immediate to conclude that our random walk model is diffusive at any temperature $\beta>0$, whenever $\alpha>4$. Moreover one also expects that the behavior of the present model is ballistic at any temperature for $\alpha \leq 2$. This follows from the very reasonable claim that a one-dimensional spin system with ferromagnetic interaction proportional to $|i-j|^{-\lambda}$ with $\lambda<1$, should have a (finite volume) non zero magnetization for all values of the temperature $\beta>0$. However, as far as we know, in the literature there is no analogous of the results contained in [11] for one-dimensional spin systems with non summable ferromagnetic interaction, since the infinite volume limit of such systems is not well defined (see e.g. [9]).

Finally, the results obtained for this bidimensional model are clearly valid also in the case $d=1$. On the other hand, a generalization to dimensions $d>2$ is also possible but it appears technically more involved. Namely, one would need to generalize the works [6] and [8] to a one-dimensional spin system in which the spin variable $\sigma_{i}$ at the site $i$ takes value in the discrete set $\left\{ \pm e_{1}, \ldots \pm e_{d}\right\}$ (the possible unit steps in a random walk in $\mathbb{Z}^{d}$ ) with $e_{i}$ being the unit vector in $d$-dimensions parallel to the $i^{\text {th }}$ axis, by proving that also for such spin systems there are two different regimes with zero magnetization and non zero magnetization.

Note that, when $d \geq 3$ such a vector spin system does not admit anymore a simple decomposition into independent Ising-type systems (i.e., with spin $\sigma=$ \pm 1 ) such as (7). E.g., for $d=3$ there are only 6 possible states for the spin at $i$ while 3 independent Ising model have $2^{3}=8$ possible states.

Acknowledgements. AP was partially supported by CNPq and FAPEMIG. RS was partially supportd by Pró-Reitoria de Pesquisa-UFMG under grant 10023. We thank Marzio Cassandro and Errico Presutti for useful discussion.

\section{References}

[1] M. Aizenman,J. T. Chayes, L. Chayes,C. M. Newman. Discontinuity of the magnetization in one-dimensional $1 /|x-y|^{2}$ Ising and Potts models, J. Statist. Phys. 50 (1988), 1-40.

[2] M. Aizenman, C. M. Newman. Discontinuity of the percolation density in one-dimensional $1 /|x-y|^{2}$ percolation models. Comm. Math. Phys. 107, no. 4, 611-647 (1986).

[3] J.P. Bouchaudi, M. Mezard, G. Parisi, J. S Yedidia. Polymers with longranged self-repulsion: a variational approach, J. Phys. A Math. Gen. 24, L1025-LI030 (1991) 
[4] P. Buttà, A. Procacci, B. Scoppola. Kac polymers, Journal of Statistical Physics 119, Nos.3/4, 643-658 (2005)

[5] S. Caracciolo, G. Parisi, A. Pelissetto. Random Walks with short-range interaction and mean field behaviour, Journal of Statistical Physics, 77, Nos. 3/4, 519-543 (1994)

[6] F. J. Dyson. Existence of a phase-transition in a one-dimensional Ising ferromagnet. Comm. Math. Phys. 12, no. 2, 91-107 (1969)

[7] A.V. Dobrynin, M. Rubinstein. Theory of polyelectrolytes in solutions and at surfaces Progress in Polymer Science 30 n.11, 1049-1118 (2005)

[8] J. Fröhlich, T. Spencer. The phase transition in the one-dimensional Ising model with $1 / r^{2}$ interaction energy. Comm. Math. Phys. 84 no. 1, 87-101 (1982)

[9] G. Gallavotti, S. Miracle-Sole. Statistical mechanics of lattice systems, Commun. Math. Phys. 5, 317-323 (1967)

[10] R. van der Hofstad, F. den Hollander, G. Slade. A new inductive approach to the lace expansion. Probability Theory Related Fields 111, 253-286 (1998)

[11] J. L. Lebowitz. Coexistence of Phases in Ising Ferromagnets, Journal of Statistical Physics, 16, No. 6, 463-476 (1977)

[12] E. Marinari, G. Parisi. On polymers with long range repulsive forces, Europhysics Letters, 15, n.7, 721-724 (1991)

[13] D. Ruelle. Statistical mechanics of a one-dimensional lattice gas, Comm. Math. Phys. 9, 267-278 (1968)

[14] H. Spohn, W. Zwerger. Decay of the Two-Point Function in OneDimensional $O(N)$ Spin Models with Long-Range Interactions, Journal of Statistical Physics, 94, Nos. 5 6, 1037-1043 (1999) 